\title{
Naturaleza jurídica de la planificación de la transmisión eléctrica
}

\section{Legal nature of electric transmission planning}

\author{
Marcelo Mardones Osorio"
}

\begin{abstract}
Uno de los cambios implementados por la Ley $\mathrm{N}^{\circ} 20.936$ fue ampliar y robustecer la planificación centralizada de los sistemas de transmisión eléctrica, a fin de asegurar su adecuado crecimiento en consonancia con las necesidades de la demanda. Dadas las particularidades de tal proceso es que el presente trabajo se aboca a dilucidar la naturaleza jurídica de la planificación de los sistemas de transmisión eléctrica, proponiendo un adecuado encuadre dogmático para facilitar su entendimiento.
\end{abstract}

Palabras clave: Infraestructuras en red, planificación, planificación centralizada, transmisión eléctrica, estudios de franja.

\section{Introducción}

Uno de los grandes tópicos de las denominadas infraestructuras en red (network industries o Netzinfrastrukturen) ${ }^{1}$ es el de su planificación o

\footnotetext{
* Abogado, Doctor y Magister en Derecho (Pontificia Universidad Católica de Chile), Máster en Asesoría Jurídica de Empresas (IE Law School, España), Profesor de Derecho Económico, Universidad de los Andes. Dirección postal: Parque central 10.620, La Reserva, Colina. Correo electrónico: marcelomardonesosorio@gmail.com.

1 En general, la doctrina nacional no ha tratado mayormente lo relativo a las infraestructuras en red, por lo que el recurso a la doctrina comparada se impone. Al respecto vid. Bickenbach 1999; Ferrari, 2000; De La Cuétara 2001, 197-275; De La Cruz 2001, 119-160; Caballero 2003; Fernandez 2003; Aguilar 2004, 375-437; Vetró 2005; López-Jurado 2009 , 759-825.
}

Artículo recibido el 12 de julio de 2019 y aceptado el 15 de mayo de 2020. 
planeamiento. En efecto, la construcción, operación y posterior desarrollo o expansión de tales infraestructuras requieren de una ordenación que permita coordinar y maximizar su utilización entre todos los interesados en su uso, de forma de alcanzar una conformación eficiente de la red. Piénsese en las infraestructuras de carreteras, en las líneas férreas, en las líneas de transmisión de energía eléctrica, en los gasoductos u oleoductos, o en los servicios sanitarios (agua potable y alcantarillado), solo por nombrar algunos. En tales casos, el transporte de carga o pasajeros, el suministro de electricidad, gas, petróleo o agua potable, así como la gestión de residuos, respectivamente, son servicios cuya prestación descansa en parte importante en la existencia de una red a través de la cual tales servicios efectivamente se materializan -de ahí que se les denomine servicios o industrias en red-2 ${ }^{2}$ la que requiere primeramente instalarse y luego expandirse, de acuerdo con los requerimientos de la demanda.

Dicha idea, que en un principio parece simple, deja de serlo si se añaden ingredientes a esa consideración inicial. Así, cabe plantearse primeramente quién debe efectuar dicha ordenación. ¿tal decisión ha de radicarse exclusivamente en el ámbito de la iniciativa privada o procede una planificación centralizada?; en este último caso, es posible aun preguntarse quién es el llamado a efectuar tal ordenación y con qué intensidad. Y, a partir de ello, interesa determinar cuál es la naturaleza jurídica de la relación que surja entre el mandante de las infraestructuras y quien las ejecuta, y, por cierto, la titularidad de estas, así como las remuneraciones que por ellas se podrá percibir.

Como se puede apreciar del tenor de las dudas esbozadas, en último término, tales interrogantes engarzan con aspectos esenciales de la ordenación económica de un país y, por coherencia, las respuestas que se planteen en el ámbito normativo deben necesariamente encontrarse en armonía con el modelo económico imperante. En dicha línea es que desde ya puede adelantarse que el tránsito desde el Estado prestacional hacia el denominado Estado garante ${ }^{3}$ impone, como modelo a seguir, ciertos límites que han de respetarse, so pena de incurrir en inconsistencias regulatorias que, en último término, pueden afectar la certeza jurídica.

El presente trabajo constituye una primera aproximación a tales cuestiones. El análisis que se efectúa comienza con una revisión del concepto "infraestructuras en red", para luego abordar la planificación y la actividad de planificación estatal, concluyendo con una referencia a la planificación en el ámbito de la transmisión de energía eléctrica, a fin de discernir en relación a su naturaleza jurídica.

\footnotetext{
2 López-Jurado 2009, 761; Montero 2016, 165.

3 Al respecto, vid. Schmidt-Assmann 2003, 257; Danós 2015, 559-571 y 566; Muñoz 2015, 2326; Moraga 2016, 2.
} 


\section{Aproximación al concepto "Infraestructuras en red"}

\section{Delimitación conceptual}

El término "infraestructuras en red" se refiere a las instalaciones físicas que son necesarias para la realización de determinada actividad o la prestación de ciertos servicios ${ }^{4}$. Tales instalaciones se caracterizan por configurarse como una "red", es decir, como una serie ordenada y concatenada de elementos que vinculan un punto de origen y de llegada. En este sentido es que el Diccionario de la Real Academia Española define "red" en su séptima acepción como un "(...) Conjunto de elementos organizados para determinado fin..." 5 . En nuestro ordenamiento es posible encontrar diversos ejemplos en los que se hace expresamente alusión a la existencia de redes, como es en materia sanitaria ${ }^{6}$, eléctrica ${ }^{7}$, telecomunicaciones ${ }^{8}$ y gas $^{9}$.

Ahora bien, la red no implica indefectiblemente la existencia de una estructura física de enlace que comunique un punto de origen y de llegada, sino que en ocasiones suele ocurrir que, existiendo un origen y un destino (denominados nodos), las estructuras de enlace no son necesariamente físicas o materiales, como ocurre por ejemplo con las rutas aéreas, marítimas o el espectro radioeléctrico en materia de telecomunicaciones. En tales casos, y pese a ello, la existencia de los referidos nodos es suficiente para, igualmente, aludir a la existencia de infraestructuras en red, en la medida que resultan de uso obligado por los operadores de un mercado, o al menos para

\footnotetext{
4 Así, de la Cuétara 2001; Aguilar 2004, 376.

5 En este sentido, vid. CABallero 2003, 148.

6 El art. 5 del D.F.L. N³82, de 1988, Ley General de Servicios Sanitarios, define servicio público de distribución de agua potable, como aquél cuyo objeto es prestar dicho servicio a través de las redes públicas exigidas por la urbanización conforme a la ley, a usuarios finales obligados a pagar un precio por dicha prestación. Y, asimismo, define servicio público de recolección de aguas servidas como aquél cuyo objeto es prestar dicho servicio, a través de las redes públicas exigidas por la urbanización conforme a la ley, a usuarios finales obligados a pagar un precio por dicha prestación. Asimismo, el art. 9 bis alude a las redes de recolección.

7 El art. 139 bis del D.F.L. N 4/20.018, fija el Texto Refundido, Coordinado y Sistematizado del D.F.L. N 1, del Ministerio de Minería, de 1982, Ley General de Servicios Eléctricos en materia de Energía Eléctrica, indica expresamente que los empalmes y medidores forman parte de la red de distribución. Iguales referencia a dicha red se encuentran en el art. 149 bis de la ley.

8 Entre muchas otras menciones, el art. 25 de la Ley N 18.168, Ley General de Telecomunicaciones, alude a las redes de servicio público telefónico y redes de servicios intermedios de telecomunicaciones.

9 El D.F.L. N³23, de 1931, Ley de Servicios de Gas, define redes de transporte como el conjunto de tuberías, equipos y accesorios destinados a transportar gas, también denominados gasoductos, que unen centros de producción o almacenamiento con redes de distribución de gas u otros centros de producción, almacenamiento o consumo (art. 2.4). Y, asimismo, define redes de distribución como el conjunto de tuberías, equipos, y accesorios, destinados a distribuir gas haciendo uso de una concesión de servicio público o de una red no concesionada hasta la salida del medidor (art. 2.5).
} 
hacerles aplicables algunas de las técnicas previstas en general para tales infraestructuras ${ }^{10}$.

La relevancia de las infraestructuras en red para el ordenamiento jurídico radica en que en muchas ocasiones ellas aportan a la conformación de oligopolios o monopolios naturales, en la medida que las referidas infraestructuras conlleven elevadas inversiones que dificulten y hagan ineficiente replicar tales redes si el mismo servicio se deseara prestar por un competidor, erigiéndose tales aspectos en barreras de entrada que ayudan a la formación de tales imperfecciones del mercado. Asimismo, la existencia de economías de escala o de densidad también pueden conllevar que los servicios prestados en red ( $y$, por ende, su infraestructura) originen tales fallas. Así, estas infraestructuras pueden erigirse en verdaderos cuellos de botella para acceder a un mercado, lo que determina que se consideren como facilidades esenciales (essential facilities), calificación que posee importantes consecuencias desde la perspectiva del acceso a tales instalaciones por terceros competidores (régimen de acceso abierto). Lo expuesto cobra aún mayor relevancia si consideramos que, en general, los servicios en red son servicios de una marcada utilidad pública y de gran relevancia económica para el país, de forma que una afectación en la prestación de los mismos incide directamente en el bienestar de los consumidores y usuarios. Estos elementos permiten afirmar que los servicios e infraestructuras en red poseen un carácter indudablemente estratégico. Pero más relevante aún, han permitido justificar la intervención del Estado en tales supuestos, y, por ende, la existencia de regulación en la materia ${ }^{11}$.

\section{Regulación de las infraestructuras en red}

En relación con las infraestructuras en red, el ámbito y por ende la intensidad de la regulación estatal puede abarcar diversos aspectos de ellas.

Así, puede referirse primeramente a la iniciativa de ejecutar tales infraestructuras, abordando los títulos necesarios para efectuar una tal instalación (libertad de entrada, autorización administrativa o concesión administrativa) y los mecanismos para poder acceder a la titularidad de la ejecución de la infraestructura (libre iniciativa privada, asignación directa o licitación pública). Asimismo, la intervención estatal por medio de la regulación puede referirse a la operación de tales instalaciones, la que puede quedar radicada en la entidad privada o en la Administración. Junto a ello, la regulación puede, por ejemplo, imponer exigencias de seguridad o calidad en la prestación del servicio, así como la fijación de precios o tarifas a cobrar por ellas. Adicionalmente, puede implicar el establecimiento de obligaciones de acceso abierto respecto de terceros competidores que requieran utilizar tales instalaciones

\footnotetext{
10 En este sentido, vid. De la Cuétara 2001; Caballero 2003, 150; Aguilar 2004, 376-377; LÓPEZ-JURAdO 2009, 765-766; MONTERO 2016, 165-166.

11 Al respecto vid. Caballero 2003, 151; Gómez-Ibánez 2003, 4-5; Manacorda y De Vincenti 2010, 23-45 y 35; Sebastiani 2010, 47-156 y 48; Montero 2016, 165-166.
} 
para poder prestar sus servicios en régimen de competencia. En última instancia, la intervención del Estado puede recaer en la extensión o ampliación de las infraestructuras en red existentes, de manera que las futuras ampliaciones se realicen en forma ordenada y eficiente.

Varios de los aspectos antes descritos pueden materializarse bajo la técnica general de regulación denominada planificación ${ }^{12}$, según se expone a continuación.

\section{La planificación}

\section{Aspectos generales}

La planificación es una técnica cuya utilización es general en los distintos ámbitos del conocimiento y que puede considerarse inevitable ${ }^{13}$. De acuerdo con el Diccionario de la Real Academia Española, el término "planificación" se define como "(...) 2. f. Plan general, metódicamente organizado y frecuentemente de gran amplitud, para obtener un objetivo determinado, tal como el desarrollo armónico de una ciudad, el desarrollo económico, la investigación científica, el funcionamiento de una industria, etc...". Como se puede apreciar, la idea de planificación se vincula con la aplicación de una metodología para el desarrollo de una determinada actividad y la consecución de ciertos objetivos predispuestos. Se trata, por tanto, de la disposición ordenada de recursos para el cumplimiento de ciertos fines preestablecidos. No se trata, por ende, de la "ordenación por la ordenación", sino que de una actividad orientada a fines cuya necesaria consecución justifica la decisión normalmente de entre varias opciones existentes.

En una economía social de mercado como la nuestra, la iniciativa económica recae prioritariamente en el sector privado, modelo el que, a priori, conduce a una adecuada asignación de los recursos. Sin embargo, existen situaciones en las cuales la libre iniciativa no siempre lleva a la decisión óptima desde el punto de vista económico. En efecto, puede ocurrir que la propia naturaleza de las infraestructuras conlleve ciertas ineficiencias que, para ser evitadas, requieren una intervención regulatoria del Estado. Tal es el caso de las infraestructuras en las que hay economías de escala o de densidad, en las que es más eficiente que solo una empresa preste el servicio en cuestión, debido a que los costos totales promedio decrecen mientras mayor es el número de usuarios del servicio. Así, repartir el área de servicio entre dos o más empresas implica que los costos totales promedios de cada una de ellas se incrementen, aumentando por ende los precios finales ${ }^{14}$. Por otra parte, la eficiencia económica del mercado no siempre es suficiente fundamento para las políticas que inciden en la asignación de recursos, existiendo otros facto-

12 Como lo sostiene LÓPEZ-JURAdo 2009, 823-824.

13 Como lo afirma Martín-Retortillo 1966, 111-146 y 121.

14 Alé 1990, 167; Mankiw 2012, 302. 
res que, de facto o de iure, pueden requerir ser satisfechos. Un ejemplo claro de ello puede verse en el ámbito de la regulación eléctrica, la que señala que el proceso de planificación de la transmisión debe considerar la planificación energética de largo plazo que desarrolle el Ministerio de Energía, así como los objetivos de eficiencia económica, competencia, seguridad y diversificación que establece la ley para el sistema eléctrico (art. 87 inc. $2^{\circ}$ de la Ley General de Servicios Eléctricos -"LGSE"-).

Dado lo anterior es que la decisión privada, que se funda esencialmente en consideraciones de maximización de beneficios, no siempre es la más adecuada, particularmente en determinados supuestos en los que existen objetivos diversos, ya de interés público ${ }^{15}$. En tales casos es que el Estado interviene en el funcionamiento del mercado, ordenando, por medio de la planificación -entre otras técnicas-, la realización de ciertas actividades.

\section{Planificación y planes}

Existe consenso en la doctrina en cuanto a que los planes son el resultado de una planificación. Así, la idea de planificación se puede vincular más bien con el procedimiento y el plan con el resultado de dicho proceso. En este sentido se ha afirmado que "(...) Planificación y Plan -actividad e instrumento- son jurídicamente conceptos concurrentes, no son, sin embargo coincidentes. Se trata de una observación que hay que tener muy en cuenta: si todo Plan en cuanto instituto jurídico, conlleva y establece siempre una determinada planificación, incluso cabe afirmar que es la forma más propia a través de la que se lleva a cabo, no es siempre cierta la proposición inversa. Se planifica a través de Planes: no solo, sin embargo, por medio de ellos..."16.

\section{La actividad de planificación del Estado}

La potestad de planificación ha sido definida como "(...) la potestad administrativa conferida a los órganos competentes para la aprobación del planeamiento de cualquier clase, fundamentalmente el relativo a la ordenación del territorio y el urbanismo, los recursos naturales, etc...." ${ }^{17}$. Como destaca la doctrina ${ }^{18}$, la actividad estatal de planificación no es novedosa, siendo una de sus formas más conocida, la planificación económica, muy propia de los años de mayor intervención estatal en la economía y hoy en día en franca retirada, habida consideración del ya innegable asentamiento a nivel mundial del modelo de mercado. Sin embargo, persiste la actividad planificadora del Estado en otras áreas específicas diversas, dado que, en rigor, la planificación puede darse respecto de cualquier tipo de actuación administrativa ${ }^{19}$, máxime si

\footnotetext{
15 En este sentido, vid. Caballero 2003, 312.

16 Martín-Retortillo 1988, 333. En este mismo sentido, vid. Moraga 2010, 131; Maurer 2012, 423.

17 MANTECA 2011, 465.

18 Oelckers 1984, 471-477; Boquera 1985, 166; Ariño 2003, 367; Caballero 2003, 308; Moraga 2010, 130; MAURER 2012, 421.

19 Martín-Retortillo 1966, 123.
} 
consideramos los principios de eficiencia y eficacia que informan el actuar administrativo de acuerdo con el art. 3 de la Ley de Bases Generales de la Administración del Estado ("LBGAE") 20 .

La actividad de planificación estatal se da en diversos sectores y no se encuentra predeterminada por la naturaleza pública o privada de los bienes que pueden sujetarse a planificación. Quizás el ámbito más conocido es el de la regulación urbanística ${ }^{21}$, pero existen diversos otros sectores en los que ella igualmente se da de manera más o menos intensa. Así ocurre, por ejemplo: (i) en el ámbito de las facultades de fiscalización, las que pueden ejercerse de forma planificada ${ }^{22}$; (ii) en el ámbito de la regulación de la energía eléctrica, particularmente en lo relativo a las instalaciones y redes de transmisión, cuya construcción y ampliación actualmente se planifican en forma centralizada; (iii) en el ámbito de las obras públicas, como es el caso de las carreteras; (iv) en materia de telecomunicaciones; ( $v$ ) en materia ambiental, como son los planes de prevención o descontaminación atmosférica, o; (vi) en el ámbito de la regulación sanitaria, por medio de los programas de desarrollo que deben presentarse y ejecutarse por los concesionarios. Sin embargo, en cada uno de tales sectores la intensidad de la intervención del Estado en la planificación es diversa.

En la actividad de planificación pueden distinguirse dos modelos ${ }^{23}$ : (i) la denominada planificación tradicional, que implica previsiones unilaterales de la Administración en relación a la oferta necesaria para satisfacer la demanda, la explotación centralizada y retribución en base a costes, modelo el que ha llegado a calificarse como contrario a la libertad de empresa; y (ii) la planificación moderna, que busca orientar la actividad privada en ciertos sectores en los que es necesario facilitar a los inversores una orientación informativo-estratégica o en sectores en los que deban asignarse recursos escasos, utilizando instrumentos que sean compatibles con el mercado y la iniciativa privada, con una explotación descentralizada y retribuciones en base a precios. En general, la planificación ha transitado desde el modelo tradicional, de imposición unilateral de planificación, a uno más bien de carácter orientativo o de fomento.

La planificación se materializa a través de diversos instrumentos de naturaleza administrativa, tanto unilaterales como contractuales ${ }^{24}$, lo que depende exclusivamente de la configuración establecida en la norma habilitante, lo que puede verse como una expresión del principio de intercambiabilidad

\footnotetext{
20 D.F.L. No 1/19.653 (2000).

21 En relación con esta materia, vid. entre muchos otros, CORDERO 2007, 269-298; FuENTES 2015, 12-21.

22 Al respecto, vid. LeAL 2015, 105-122.

23 Ariño 2003, 372-373. Asimismo, vid. Raschauer 2009, 243-244; Maurer 2012, 424.

24 MAURER 2012, 424-425. Y ello es fácilmente comprensible dado que, como destaca la doctrina, tanto el contrato como el acto "(...) son, en un Estado de Derecho, dos modalidades de ejecución de la norma para la realización de los fines del Estado...". ARIÑo 2007, 79-102 y 86.
} 
de las técnicas administrativas, en cuya virtud, en determinados casos, la Administración puede lograr una misma finalidad mediante diferentes tipos de actuaciones ${ }^{25}$. Así, por ejemplo, en el ámbito de las obras públicas, la planificación se materializa a través de concesiones o contratos de obra pública. En el ámbito ambiental, los planes de prevención o descontaminación se materializan a través de actos administrativos, esto es, actos unilaterales que tienen la forma de decreto supremo. En materia eléctrica, la planificación se materializa por medio de actos administrativos unilaterales, esto es, decretos supremos que aprueban los planes de expansión previamente elaborados por la Comisión Nacional de Energía ("CNE").

Por otra parte, si bien la planificación suele estar dotada de altos grados de discrecionalidad, ella no es absolutamente autónoma -de hecho, como es sabido, nunca lo es-. Normalmente, la planificación responde a ciertas exigencias, criterios o límites preestablecidos por la ley, a los cuales ella debe necesariamente ceñirse. $Y$ es solo al interior de tales limites que la Administración puede planificar en forma válida.

\section{La planificación de las infraestructuras en Red. Especial referencia a la planificación de la transmisión eléctrica}

\section{Aspectos generales}

Dados los impactos que para la economía y el mercado poseen las infraestructuras en red es que su planificación reviste una importancia fundamental. Como hemos adelantado, la actividad estatal de planificación puede manifestarse con diversas intensidades según sea el sector en cuestión. Así, puede contemplarse desde la perspectiva de la ejecución inicial de una determinada infraestructura, o en forma posterior, respecto de la extensión y ampliación de una estructura existente, sea que ella se realizó por iniciativa privada o fue objeto de planificación ab initio. En efecto, en ciertos sectores estratégicos, la ejecución de determinadas instalaciones, así como la decisión relativa a su expansión, requiere de la intervención del Estado. La referida intervención usualmente busca asegurar que el servicio en cuestión pueda prestarse de la forma más eficiente posible, alcanzando al mayor número de usuarios del servicio de que se trate considerando la demanda existente y proyectada, así como, en la medida de lo posible, que el servicio se preste en régimen de competencia o cuando menos bajo un régimen que intente emular la existencia de tal competencia.

Desde luego, es solo el carácter estratégico del servicio -en los términos expuestos supra I.1- el que justifica una mayor intensidad en la actividad planificadora del Estado, debiendo en caso contrario radicarse con carácter general en la iniciativa privada las decisiones de inversión y su planificación.

25 En relación al referido principio vid. Diez-PICAzo 1982, 7-46 y 21; VILLAR 1999, 40; En Chile, vid. LEAL 2015a, 15-16. 


\section{Coordinación de la planificación}

Un reto importante de la planificación de las infraestructuras en red es su integración y coordinación con otras ordenaciones. En efecto, la planificación de las redes requiere un importante grado de coordinación con otros mecanismos, de entre los que más relevancia posee es la denominada ordenación o planificación del territorio, que importa no solo la coordinación de factores territoriales con económicos y sociales, sino que asimismo la armonización de diversos niveles de administración territorial, entre ellos, y con la iniciativa privada ${ }^{26}$. Precisamente, las infraestructuras en red descansan de manera relevante en el uso del territorio ${ }^{27}$.

En el ámbito urbano, el art. 28 decies de la Ley General de Urbanismo y Construcción ("LGUC"), denominado "Transparencia en el ejercicio de la potestad planificadora", señala que la planificación urbana es una función pública cuyo objetivo es organizar y definir el uso del suelo y las demás normas urbanísticas de acuerdo con el interés general ${ }^{28}$. La relevancia de esta planificación es sustancial. Cabe recordar, como lo hace la más autorizada doctrina, que "(...) el ius aedificandi urbano ha dejado de ser una facultad libre del propietario para convertirse en una determinación pública realizada precisamente por el Plan urbanístico; el propietario no tiene otras posibilidades edificatorias sobre el suelo que las que el Plan le otorga -puede no otorgarlas-, y en la medida precisa en que lo hace, con todas las determinaciones relevantes, salvo las del simple diseño arquitectónico..."29. Así, la planificación urbana puede imponer severas restricciones a las posibilidades de ejecución de infraestructuras en red.

A partir de tal idea y con ánimo de coordinar los diversos mecanismos de planificación sectorial, el mismo art. 28 decies, letra e), señala que el ejercicio de la potestad planificadora deberá ser consistente, entre otros, con los estudios técnicos referidos, entre otros, a infraestructura sanitaria y energética, conforme establezca la Ordenanza General de Urbanismo y Construcciones ("OGUC"), los que necesariamente deberán estar en coordinación con las políticas sectoriales asociadas a cada materia. De acuerdo con el art. 2.1.24 OGUC, uno de los seis tipos de usos de suelo que se reconocen es "infraestructura", el cual, según el art. 2.1.29 OGUC, abarca las edificaciones o instalaciones y las redes o trazados destinados a la infraestructura de transporte (tales como vías y estaciones ferroviarias, terminales de transporte terrestre, recintos marítimos o portuarios, instalaciones o recintos aeroportuarios, etc.), sanitaria (tales como plantas de captación, distribución o trata-

\footnotetext{
26 Pérez 1998, 97-138.

27 Con relación al ordenamiento territorial y la regulación eléctrica, vid. MARTínez 2017, 103-117.

28 Como señala la doctrina, "(...) Por planeamiento territorial y urbanístico se entiende aquella operación técnica con reflejo documental por la cual se anticipa la utilización y usos que ha de darse a un determinado territorio; de esta forma también se concreta el alcance del derecho de edificar. El planeamiento territorial lo es a escala más amplia y el urbanístico a escala más reducida, normalmente a partir del ámbito municipal...". PARADA 2010, 343.

29 García De Enterría y Fernández 2006, 158.
} 
miento de agua potable o de aguas servidas, de aguas lluvia, rellenos sanitarios, estaciones de transferencia de residuos, etc.), y energética (tales como centrales de generación o distribución de energía, de gas y de telecomunicaciones, gasoductos, etc.). El mismo artículo añade que, para estos efectos, se entenderá por redes y trazados, todos los componentes de conducción, distribución, traslado o evacuación, asociados a los elementos de infraestructura. Y, asimismo, señala que las redes de distribución, redes de comunicaciones y de servicios domiciliarios y en general los trazados de infraestructura se entenderán siempre admitidos y se sujetarán a las disposiciones que establezcan los organismos competentes ${ }^{30}$.

Desde la perspectiva rural, hay que considerar la existencia de los Planes Regionales de Ordenamiento Territorial ("PROT"), incorporados en la Ley Orgánica Constitucional sobre Gobierno y Administración Regional ("LOCGAR") por la Ley $N^{\circ} 21.074$, de 2018. Los PROT se definen como instrumentos que orientan la utilización del territorio de la región para lograr su desarrollo sustentable a través de lineamientos estratégicos y una macro zonificación de dicho territorio (art. 17 a) LOCGAR). La relevancia de los PROT para las infraestructuras en red radica en que ellos establecen condiciones para la localización de las infraestructuras en zonas no comprendidas en la planificación urbanística, identificando las áreas para su localización preferente. Cabe señalar que las referidas condiciones son de carácter imperativo y su incumplimiento se sanciona con la caducidad de las autorizaciones respectivas ${ }^{31}$.

Es dable indicar que los PROT cuentan con las siguientes limitaciones en su ámbito de competencias:

(i) No pueden regular materias que tengan un ámbito de influencia u operación que exceda del territorio regional, abarcando por tanto dos o más regiones o incluso con un ámbito nacional, como ocurre precisamente con la planificación de la transmisión eléctrica. Sin embargo, el citado art. 17a) señala que la política nacional de ordenamiento territorial ("PNOT"), Ilamada a contener principios, objetivos, estrategias y directrices sobre la materia, contendrá asimismo las reglas aplicables a las redes e infraestructuras que tengan un ámbito de influencia u operación que exceda al territorio regional, generando una serie de incertidumbres en cuanto a la relación de dicha política con las reglas antes descritas de la OGUC en materia de infraestructuras $^{32}$. En la versión de la PNOT sometida a consulta pública durante el

30 Así, como ha señalado la CGR en su Dictamen $N^{\circ} 36.448 / 2016$, "(...) el reseñado artículo 2.1.29. regula en forma diversa el uso de suelo infraestructura según se trate de 'redes o trazados', los que se entienden siempre admitidos, o de 'instalaciones o edificaciones' necesarias para ese uso, que no formen parte de la red, respecto de las cuales los instrumentos de planificación pertinentes pueden establecer condiciones o requisitos que permitan su emplazamiento (aplica el criterio contenido en el dictamen N 94.146, de 2014, de esta entidad de control)...".

31 Nótese que la sanción de caducidad se relaciona solo con las autorizaciones. Por tratarse de una sanción, ella debe interpretarse en forma restrictiva, de manera que ella no alcanza a otros títulos, como por ejemplo, las concesiones.

32 Así, Martínez 2017, 109-110. 
año 2017 no se incluyó lo relativo a las referidas reglas. Sin embargo, en los trabajos reglamentarios actualmente en desarrollo, se impone la idea de que las referidas reglas aplicables son las ya existentes en los ordenamientos sectoriales respectivos, de manera que en este punto la PNOT no incorporaría mayores innovaciones.

(ii) Tampoco pueden regular áreas que estén sometidas a planificación urbanística, es decir, a cualquiera de los instrumentos de planificación territorial, los que, en los ámbitos de acciones que les compete, definen los usos de suelo de cada zona (art. 2.1.24 OGUC) ${ }^{33}$.

\section{Planificación de la transmisión eléctrica}

\section{a) Consideraciones generales sobre la transmisión eléctrica}

Desde la dictación de la LGSE, la transmisión ha sido uno de los segmentos que mayores modificaciones normativas ha vivido, fruto de su relevancia para el mercado y sistema eléctrico. En esta materia es muy útil lo señalado por el Panel de Expertos en su Dictamen No 1-2013, en relación al sistema de transmisión troncal existente antes de la entrada en vigencia de la Ley $N^{\circ} 20.936$, pero igualmente aplicable a la actualmente denominada transmisión nacional. En éste, dicha instancia sostuvo:

"(...) A partir de la Ley 19.940 se establece, mediante normas de orden público, una nueva regulación del servicio de transporte de electricidad. Contempla este ordenamiento un sistema de transporte para cada sistema interconectado, constituido por instalaciones del sistema de transmisión troncal, de subtransmisión y adicional. Como columna vertebral del sistema de transporte, el sistema troncal concentra los mayores ámbitos de regulación, que van desde su calificación de servicio público y la exigencia de desintegración vertical en su propiedad, hasta los mecanismos para proceder a la expansión de las instalaciones, la valorización de las mismas y la tarificación por su uso. Todo ello se somete a procedimientos administrativos y contenciosos especiales con participación activa de las empresas eléctricas de los diversos segmentos, los propietarios y usuarios del sistema troncal, los órganos técnicos de cada CDEC y la Comisión Nacional de Energía..."

Según consta en la historia de la Ley Corta ${ }^{34}$, con anterioridad a dicha ley, el mecanismo de tarificación no operaba correctamente, pues el transmisor recibía ingresos que le permitían cubrir sus costos totales con independencia de las saturaciones o situaciones de escasez del sistema, lo que los desincentivaba a invertir en nuevas instalaciones, provocando importantes

\footnotetext{
${ }_{33}$ Sin perjuicio de lo anterior, cabe tener presente que los PROT a los que se refiere la Ley $N^{\circ} 21.074$ solo podrán aprobarse por los gobiernos regionales una vez que entren en vigencia la PNOT y el reglamento de los PROT, establecidos en el párrafo quinto del literal a) del art. 17 de la LOCGAR.

34 Historia de la Ley N¹9.940, de 2004.
} 
cuellos de botella en el sistema. Por otra parte, la legislación no consideraba a la transmisión como un "servicio público", por lo que no existía obligación de los propietarios de la red para expandir el sistema.

Tras la dictación de la Ley Corta I, tales falencias fueron resueltas, si bien la cada vez más creciente relevancia del sistema de transmisión troncal para el adecuado abastecimiento de la demanda impuso la necesidad de una visión estratégica de largo plazo, en la que, además, debía incluirse la subtransmisión -ahora denominada transmisión zonal-, como segmento sujeto a planificación obligatoria, asegurando así un desarrollo integral de la red ${ }^{35}$. Y junto a ello, la nueva regulación se propuso incorporar criterios de coordinación entre los intereses energéticos y los diversos intereses tutelados por medio de la planificación del ordenamiento territorial, de forma que no solo fueran consideraciones económicas las que guiaran la decisión de planificación de la transmisión.

\section{b) Régimen general de planificación de la transmisión}

Como adelantamos, el régimen de planificación de la transmisión fue sometido a una modificación sustancial por medio de la Ley $N^{\circ} 20.936$, que tuvo por objeto establecer un nuevo régimen de planificación "(...) desarrollada por la autoridad de manera vinculante, para permitir el desarrollo de proyectos de transmisión que generen un beneficio nacional que vaya más allá de la simple reducción de los costos operacionales de corto plazo del sistema eléctrico, permitiendo la reducción de barreras de entrada, eliminando desacoples económicos entre distintas zonas del país y facilitando una mayor incorporación de las energías renovables que el país posee en alto potencial..." ${ }^{36}$.

El nuevo esquema de planificación de la transmisión descansa sobre los siguientes aspectos esenciales:

(i) Se trata de una planificación centralizada de carácter vinculante que puede abarcar la práctica totalidad de segmentos que integran el sistema de transmisión, salvo los sistemas de interconexión internacional, que cuentan con un régimen especial de expansión ex art. 99 bis LGSE.

(ii) La planificación abarca tanto obras nuevas como de ampliación, ambas agrupadas bajo el concepto genérico de "obras de expansión" 37 .

\footnotetext{
35 Historia de la Ley N²0.936, de 2016.

36 ĺdem.

37 De acuerdo con el art. 89 LGSE, son obras de ampliación aquellas que aumentan la capacidad o la seguridad y calidad de servicio de líneas y subestaciones eléctricas existentes, añadiendo que no se consideran como obras de ampliación aquellas inversiones necesarias para mantener el desempeño de las instalaciones conforme a la normativa vigente. El mismo artículo indica que se entenderá por obras nuevas aquellas líneas o subestaciones eléctricas que no existen y son dispuestas para aumentar la capacidad o la seguridad y calidad de servicio del sistema eléctrico. Como puede verse, la distinción entre obras nuevas y de ampliación descansa en las ideas fundamentales de existencia de la instalación y complementariedad de la obra de ampliación, determinada de acuerdo con criterios técnicos.
} 
(iii) Se trata de una planificación de carácter anual, preparada por la CNE y fijada por el Ministerio de Energía por decreto supremo exento. Así, la planificación es fruto de un procedimiento administrativo reglado tramitado principalmente ante la CNE, conformado por los siguientes hitos fundamentales (art. 91 LGSE): (a) propuesta de expansión por el Coordinador Eléctrico Nacional; (b) presentación de propuestas de proyectos de expansión por los privados a la CNE; (c) emisión de un informe técnico preliminar ("ITP") por la CNE; (d) presentación de observaciones por los interesados respecto del ITP; (e) emisión del informe técnico final ("ITF") del plan de expansión anual por la CNE, aceptando o rechazando fundadamente las observaciones planteadas; $(f)$ presentación de discrepancias ante el Panel de Expertos; (g) resolución del Panel; y, (h) corrección, en su caso, del ITP, que pasa a llamarse informe técnico definitivo ("ITD") y envío al Ministerio de Energía para la emisión del o los decretos aprobatorios del plan de expansión correspondiente al año respectivo.

(iv) A partir de dicho hito, la LGSE divide el procedimiento administrativo diferenciando entre obras de ampliación y obras nuevas: mientras las primeras deben aprobarse por decreto dentro de un plazo de 15 días desde la recepción del ITD en el Ministerio, en las segundas debe diferenciarse entre aquellas obras nuevas que requieren la realización de un estudio de franja, de aquellas que no. Este decreto, con tal distinción, debe ser emitido dentro de un plazo de 60 días, desde la recepción del ITD en el Ministerio (art. 92 LGSE).

Tratándose de las obras de expansión que no requieren de estudio de franja, ellas deben ser objeto de licitación pública internacional por el Coordinador Eléctrico Nacional dentro de un plazo de 12 meses desde la vigencia del respectivo decreto -i.e., desde su publicación-, para procederse a adjudicar los derechos de ejecución y explotación de las obras respectivas, por medio de un decreto del Ministerio de Energía. Este decreto es el que constituye los derechos de ejecución de las obras de expansión y solo con él surgen tales derechos, como se desprende del art. 122 LGSE.

Mientras que, tratándose de las instalaciones que sí requieren la realización del referido estudio, en ese mismo plazo debe iniciarse la licitación del estudio en cuestión. Una vez finalizado el mencionado procedimiento y habiéndose dictado por el Ministerio el decreto que fija la franja preliminar, se retoma el procedimiento general antes descrito, debiendo el Coordinador Eléctrico Nacional realizar la licitación pública internacional de las obras para luego procederse a la adjudicación de los derechos de ejecución y explotación de las obras en cuestión.

\section{c) Algunas consideraciones sobre los estudios de franjas}

La regulación de las franjas incluida por la Ley $\mathrm{N}^{\circ} 20.936$ tuvo por objeto hacer frente a las dificultades que los proyectos de transmisión estaban encontrando, esencialmente en términos de relacionamiento con las comunidades afectadas. En general, la regulación de franjas lo que busca es poder 
determinar de manera participativa el mejor trazado para ciertos proyectos de transmisión. Así, en el Mensaje de la Ley N²0.936 se calificó al estudio de franja como "(...) una propuesta que busca lograr equilibrio económico-social-ambiental en la definición de trazados; dota al sistema de mayores grados de certeza para la realización de los proyectos; asigna al Estado un rol central en la evaluación de trazados; reduce los riesgos de las empresas licitantes y operadoras, con el objeto de reducir las tarifas eléctricas; y da legitimidad al desarrollo de proyectos de transmisión ante la opinión pública y las comunidades..."38.

El modelo de franjas implementado en nuestro país implica una planificación público-privada pero con alta injerencia estatal, siguiendo así los modelos existentes en Colombia, Estados Unidos, Australia y Suiza, en los que el Estado cuenta con organismos con capacidades de planificación y desarrollo, y los recursos necesarios para llevar o acompañar los procesos de planificación y de definición de franjas o trazados ${ }^{39}$.

El estudio de franja y la determinación de la franja respectiva se encuentra parcialmente regulada en la LGSE, que establece algunos criterios que pueden considerarse en la fijación de los trazados respectivos, tales como los niveles de tensión de las instalaciones, el propósito de uso, las dificultades de acceso a o desde polos de desarrollo de generación, la complejidad de su implementación y la magnitud de las mismas, todo ello, de acuerdo con lo dispuesto en el reglamento, contenido en el D.S. N 139/2016, de Energía, reglamento para la determinación de franjas preliminares para obras nuevas de los sistemas de transmisión ("reglamento de franjas"). Tales criterios pueden agruparse bajo dos consideraciones esenciales: (i) sostenibilidad y (ii) reducción de riesgos en la ejecución de los proyectos ${ }^{40}$.

En dicho contexto es que la realización del estudio se ciñe a la metodología EPRI-GTC (Electric Power Research Institute y Georgia Transmission Corporation) ${ }^{41}$ utilizada en los Estados Unidos, pero incorporada en nuestra regulación con diversas modificaciones considerando la realidad nacional. En virtud de tal metodología, el estudio de franjas implica la determinación inicial de un área general (área preliminar de estudio) a partir de la cual se depuran en forma progresiva diversas opciones de trazado (área de estudio de franjas, corredores alternativos y franjas alternativas) hasta llegar a la denominada franja alternativa, de acuerdo con los análisis que se vayan efectuando, todo ello bajo una "lógica de embudo"42.

En cuanto al procedimiento, la LGSE desarrolla de manera un tanto confusa las diversas etapas que lo componen, las que se pueden resumir de

\footnotetext{
38 Historia de la Ley $N^{\circ} 20.936$, de 2016.

39 Ídem.

40 Como se da cuenta en Ministerio de Energía 2018, 25-26.

41 Desarrollada por HOUSTON y JOHNSON 2006.

42 Ministerio de Energía 2018, 33-34.
} 
la manera que sigue -si bien a la fecha aún no se materializa íntegramente al menos un procedimiento-:

(i) Una vez publicado el decreto que fija las obras nuevas, el Ministerio iniciará el estudio de franja, para lo cual elaborará unas bases técnicas y administrativas y procederá a licitar la ejecución del mismo, actuando la SEC como organismo técnico asesor. El referido estudio se financia a partir de un presupuesto anual elaborado por la Subsecretaría de Energía, solventado por medio del cargo por servicio público a que alude el art. 212-13 LGSE. Si bien la ley no lo explicita, lo cierto es que dichas sumas no ingresan al erario nacional, por cuanto su origen no es fiscal -el referido cargo lo paga la demanda-, sin perjuicio de que su administración la efectúe la referida Subsecretaría por medio de una o más cuentas extrapresupuestarias ${ }^{43}$.

(ii) Durante el desarrollo del estudio de franjas, se ejecutará en forma paralela: (i) el procedimiento de evaluación ambiental estratégica ("EAE") ${ }^{44}$, a fin de entregar orientaciones y lineamientos para definir y evaluar las franjas alternativas como para seleccionar posteriormente una de ellas, permitiendo focalizar el proceso de decisión en aquellos elementos que sean clave para definir distintas franjas alternativas, actuar de manera preventiva y considerar desde etapas tempranas aspectos de sustentabilidad que pueden incidir en la localización de una línea de transmisión ${ }^{45}$; y (ii) un proceso de participación o consulta indígena en conformidad con las previsiones del Convenio N ${ }^{\circ} 169$ de la Organización Internacional del Trabajo ("OIT") y lo dispuesto en el D.S. $N^{\circ}$ 66/2013, del Ministerio de Desarrollo Social, que aprueba el reglamento que regula el procedimiento de consulta indígena en virtud del artículo 6 $\mathrm{N}^{\circ} 1$ letra a) y $\mathrm{N}^{\circ} 2$ del referido Convenio, si bien no existe una regulación específica para la participación indígena (art. 93 LGSE).

(iii) Finalizada la $E A E$, el procedimiento del estudio de franjas concluye con la emisión de un informe que contendrá una propuesta de franja, denominada franja alternativa, la que es sometida a aprobación del Consejo de Ministros para la Sustentabilidad, tras la cual el Ministerio de Energía debe emitir un decreto supremo exento fijando la denominada franja preliminar. En dicho decreto el Ministerio puede establecer que los predios afectados por la referida franja preliminar podrán ser gravados con una o más servidumbres de aquellas señaladas en los arts. 50 y siguientes de la LGSE. Como se puede apreciar, en dicho decreto no se perfecciona el gravamen, sino que solo se identifican aquellos que en forma posterior podrán constituirse de pleno

\footnotetext{
43 Las cuentas extrapresupuestarias pueden existir solo si así lo autoriza la ley (art. 4 del D.L. N¹.263/1978, Ley Orgánica de Administración Financiera del Estado) o la Contraloría General de la República (de acuerdo con sus atribuciones constitucionales en materia de contabilidad general de la Nación). Al respecto, vid. PAlLAVIcINI 2015, 69-70. Hoy en día, tales cuentas no han sido autorizadas y las sumas recaudadas se incorporan en el presupuesto del Ministerio de Energía, como se adelantó, de forma incorrecta.

44 Al respecto, vid. Ministerio de Energía, Ministerio del Medio Ambiente y Servicio de Evaluación AmBIENTAL 2018.

45 Ministerio de Energía 2018, 33.
} 
derecho de acuerdo con lo señalado por el art. 97 LGSE, esto es, una vez que el adjudicatario de los derechos de ejecución y explotación del proyecto de obra nueva defina el trazado y cuente con la correspondiente RCA para la ejecución del proyecto. En tal caso, trátase por tanto de un supuesto de constitución administrativa de servidumbres legales, las que son una especie de limitación al dominio ${ }^{46}$. Precisamente, la norma alude a que el gravamen es "por causa de utilidad pública", explicitando así cuál es el específico aspecto de la función social del dominio que fundamenta la limitación que tales gravámenes importan. Se ha señalado que la utilidad pública "(...) significa beneficio colectivo -referido especialmente al orden interior de la República-, no tiene significación patrimonial, ni protege intereses de personas o grupos..." ${ }^{47}$. La principal consecuencia de lo expuesto es que los gravámenes que se impongan por causa de utilidad pública para permitir la materialización de la línea de transmisión en la franja, generan el derecho a la indemnización de los daños y perjuicios que con ello se generen ${ }^{48}$. El decreto es objeto de publicidad en el diario oficial, y a partir de tal publicación quienes puedan verse afectados por las eventuales servidumbres se entenderán notificados (notificación ficta). Sin embargo, la LGSE no explicita la finalidad de tal notificación ficta, dado que en este procedimiento especial de constitución de servidumbres eléctricas no se contempla la posibilidad de presentar oposiciones, como sí se prevé en el régimen general. En todo caso, la posibilidad de imponer el referido gravamen está sujeto a un plazo de caducidad de 5 años, desde la dictación del decreto antes aludido, prorrogables por causa justificada por una sola vez por hasta 2 años (art. 94 LGSE).

(iv) Ya identificada la franja preliminar, el Coordinador Eléctrico Nacional debe proceder a licitar la ejecución de las obras en cuestión y a la adjudicación respectiva, si bien la fijación de los derechos de ejecución se realiza por el Ministerio de Energía por medio de decreto supremo.

(v) El titular de los derechos para la ejecución de la obra debe realizar la evaluación ambiental del proyecto, determinando el trazado específico a seguir al interior de la franja preliminar, y obtener la correspondiente RCA favorable.

\footnotetext{
46 Sentencia TC Rol No 2.751-14-INA (2015).

47 Verdugo, Pfeffer y Nogueira 2005, 311-312; Fernández 2014, 115-121 y 116.

48 Como señala MAYER, "(...) El derecho más importante del propietario que sufre las consecuencias de la restricción a la propiedad por causa de utilidad pública, y que al mismo tiempo es un verdadero derecho subjetivo público, es el de obtener una indemnización...". MAYER 1951, 318-319. En este sentido se ha pronunciado el TC en la antes citada sentencia Rol N N 2.751-14INA (2015), al señalar en relación con la constitución administrativa de servidumbres eléctricas, que "(...) tal como expresara esta Magistratura en sentencias roles $N^{\text {os }} 245$ (considerando $41^{\circ}$ ) y 1669 (considerando $77^{\circ}$ ), estas servidumbres otorgan al dueño del predio sirviente el derecho a que se le paguen los perjuicios que la ley señala (artículo 69, Nos 1 al 3), cuya evaluación incluso puede ser objeto de acuerdo con el concesionario. Son justamente estas reparaciones e indemnizaciones, que el concesionario debe pagar al dueño del predio sirviente, lo que armoniza los derechos de uno y otro, sin desequilibrios arbitrarios y sin que sea menester sacrificar a ninguno en o beneficio de la colectividad (según ordena el artículo $1^{\circ}$, inciso cuarto, constitucional)..."
} 
(vi) Obtenida la RCA, ella debe presentarse ante el Ministerio de Energía, el que procederá a dictar un decreto exento en el que se determina el trazado definitivo y la franja de seguridad asociada a dicho trazado, constituyéndose, por el solo ministerio de la ley, servidumbre eléctrica sobre la referida franja de seguridad, la que obviamente abarca el trazado. El referido decreto se dicta previo informe de la SEC respecto a que la información entregada por el titular del proyecto respecto de las obras y servidumbres se ajusta a la normativa eléctrica vigente. La norma añade que el titular del proyecto será considerado titular de concesión eléctrica para los efectos del arts. 31 bis y 34 bis de la LGSE. Todo lo expuesto es sumamente relevante ya que permite colegir que, en el marco del procedimiento de franjas, no hay necesidad de contar con una concesión eléctrica a partir de la cual surja una servidumbre, sino que la servidumbre surge ex ministerio legis, sin necesidad de otro título, sin perjuicio de los trámites posteriores necesarios para su materialización. Precisamente por ello, esto es, dada la inexistencia de un título concesional, la LGSE (art. 97) señala que el titular del proyecto será considerado concesionario, tratándose así de un concesionario ficto.

(vii) Asimilando la tramitación a la de una concesión eléctrica, el reglamento de franjas añade que dentro de los 30 días siguientes a la publicación del decreto que fija el trazado definitivo, el titular lo deberá reducir a escritura pública, debiendo, asimismo, a partir de la fecha de reducción, iniciar las gestiones para hacer efectivas las servidumbres legales conforme a los arts. 62 y siguientes de la LGSE, i.e., solicitud de designación de comisión tasadora, solicitud judicial de posesión material, consignación de indemnización y toma de posesión material.

\section{d) Limitaciones legales impuestas a la planificación de la transmisión}

Como adelantamos, la planificación no es un proceso absolutamente discrecional, sino que, muy por el contrario, la LGSE establece una serie de exigencias que han de cumplirse durante el proceso de formulación del plan de expansión, so pena de incurrir en una infracción discrepable ante el panel de expertos o anulable judicialmente si no fue objeto de discrepancia. Así, en primer lugar, la planificación de la transmisión debe "considerar" 49 una serie de aspectos para ser válidamente ejecutada, a saber:

(i) La planificación energética de largo plazo ("PELP") elaborada por el Ministerio de Energía ${ }^{50}$, si bien actualmente no existe claridad en relación a la

\footnotetext{
49 Existen innumerables ocasiones en las que tanto la LGSE como sus diversos reglamentos establecen la obligación de "considerar" algo, lo que ha introducido importantes cuotas de incerteza, al no precisarse en qué consiste tal "consideración".

50 Como consta en el Mensaje con que se acompañó la actual Ley $N^{\circ} 20.936$, p.10, la PELP consiste en un proceso quinquenal de planificación energética para un horizonte de 30 años, llamado a entregar los lineamientos generales relacionados con escenarios de desarrollo del consumo (demanda) y de la oferta de energía eléctrica que el país podría enfrentar en el futuro, para lo que se incorpora una prospectiva respecto de las tecnologías de generación disponibles, su evolución y desarrollo; así como eventuales nuevas alternativas tecnológicas para el debido
} 
real extensión del deber de que el plan de expansión de la transmisión deba "considerar" los escenarios de la PELP, ya que si bien tal cuestión se planteó ante el Panel de Expertos en las discrepancias que se resolvieron por el dictamen $\mathrm{N}^{0} 7 / 2018^{51}$, ellas no se zanjaron por no encontrarse vigente la PELP al momento de elaborarse el referido plan de expansión. Tal cuestión está siendo objeto de clarificación en el actual borrador sometido a consulta pública del reglamento de los sistemas de transmisión y de la planificación de la transmisión (art. 70), al señalar que "(...) La Comisión deberá ajustar cada uno de los Escenarios Energéticos definidos por el Ministerio, definiendo la capacidad de expansión de generación y su localización en las distintas barras del Sistema Eléctrico..." [las cursivas son nuestras].

(ii) Los objetivos de eficiencia económica, competencia, seguridad y diversificación que establece la ley para el sistema eléctrico, los que, por cierto, no se encuentran dispuestos en forma clara en la regulación, sino que se desprenden de diversas disposiciones de la LGSE.

(iii) Las holguras o redundancias necesarias para incorporar los objetivos de la transmisión indicados previamente, de acuerdo con los criterios señalados infra y los aspectos metodológicos establecidos en el reglamento. En el actual borrador sometido a consulta pública del reglamento de los sistemas de transmisión y de la planificación de la transmisión (art. 62) se señala que "(...) deberá entenderse por planificación con holgura aquella en la que se otorga un dimensionamiento superior al requerido para la obra de transmisión analizada de manera que permita cubrir la necesidad de expansión del sistema frente a diferentes escenarios y se justifique económicamente. Para ello, la Comisión podrá proponer instalaciones de transmisión con un estándar constructivo mayor al de energización, proponer líneas de transmisión con torres para al menos dos circuitos, subestaciones con mayor cantidad de posiciones para futuras conexiones, entre otros. Se entenderá por planificación con redundancia la incorporación de aquella infraestructura que permita enfrentar la pérdida o falla de un elemento de transmisión para cumplir con el abastecimiento de la demanda, de acuerdo a lo que señale la normativa técnica vigente. Adicionalmente, para efectos de la aplicación del criterio de redundancia, la Comisión podrá definir los proyectos de expansión que permitan cumplir con los objetivos de la Ley con el nivel de seguridad requerido para ello y que sean económicamente eficientes para el sistema [...]. Los criterios de holgura y redundancia podrán aplicarse durante las distintas etapas del proceso de planificación de acuerdo a lo señalado en los artículos

\footnotetext{
abastecimiento de la demanda. Por otro lado, también debiera entregar lineamientos sobre el comportamiento del consumo, incluyendo políticas de eficiencia energética, promoción de nuevas tecnologías, generación distribuida, nuevos tipos y formas de consumos, redes inteligentes, entre otros. Dicha planificación quinquenal puede ser actualizada en forma anual (art. 83 LGSE). El detalle del procedimiento de planificación energética se contiene en el D.S. N 134/2016, de Energía, que aprueba reglamento de planificación energética de largo plazo.

51 Dictamen Nº 7/2018 (2018).
} 
siguientes, debiendo la Comisión justificarlos debidamente en cada caso en el informe técnico que fija el Plan de Expansión..." ${ }^{52}$.

(iv) La información sobre criterios y variables ambientales y territoriales disponible al momento del inicio de éste, incluyendo los objetivos de eficiencia energética que proporcione el Ministerio de Energía. Trátase del denominado informe "VAT", que es remitido todos los años por el Ministerio a la CNE dentro del primer trimestre del año en curso.

(v) La participación ciudadana por medio de la realización de observaciones en el proceso respectivo por quienes se incorporen en el registro de participación ciudadana que lleva la CNE.

Junto a ello, de acuerdo con la LGSE, para alcanzar los objetivos de eficiencia económica, competencia, seguridad y diversificación del sistema eléctrico, deben considerarse una serie de criterios en el plan de expansión respectivo ${ }^{53}$ : (i) minimización de los riesgos en el abastecimiento; (ii) creación de condiciones que promuevan la oferta y faciliten la competencia, propendiendo al mercado eléctrico común -esto es, aquél que surge con ocasión de la interconexión de los demás segmentos de la transmisión-, para el abastecimiento de la demanda a mínimo costo con el fin último de abastecer los suministros a mínimo precio; (iii) instalaciones económicamente eficientes y necesarias para el desarrollo del sistema, en los distintos escenarios energéticos que defina el Ministerio en la PELP, y; (iv) posibles modificaciones de instalaciones de transmisión existentes que permitan realizar las expansiones necesarias de manera eficiente.

Como señala el art. 72 del borrador de reglamento de los sistemas de transmisión y de la planificación de la transmisión, el cumplimiento de los antes citados objetivos y criterios se materializa por medio de la aplicación de la metodología que desarrolla dicho reglamento, la que tiene como resultado el plan de expansión. La metodología considera las siguientes fases: (i) análisis preliminar; (ii) análisis de suficiencia y eficiencia operacional; (iii) análisis de seguridad y calidad de servicio; (iv) análisis de factibilidad técnica de los proyectos de expansión; (v) análisis económico de los proyectos de expansión; (vi) análisis de resiliencia; (vii) análisis de mercado eléctrico común; y (viii) conformación del plan de expansión.

\footnotetext{
52 En el Mensaje de la Ley $\mathrm{N}^{\circ} 20.936$ consta que las holguras permiten responder ante escenarios desajustados a menor costo que con un sistema de transmisión ajustado, así como la integración técnica y económica, y facilitan el financiamiento de proyectos de generación alejados de los centros de consumo; asimismo, las holguras permiten una mayor competencia en el sector generación lo que redunda en una baja en los costos marginales y en los costos de operación de largo plazo del sistema, beneficios que son traspasables al consumidor final; y por último, no solo reducen el costo esperado de operación futuro, sino que reducen notablemente la dispersión de los costos marginales y, con ello, el riesgo que enfrentan todos los proyectos de generación. Historia de la Ley $N^{\circ} 20.936$, de 2016.

53 En este sentido, vid. el Dictamen $N^{\circ} 7 / 2018$.
} 
e) Naturaleza jurídica del vínculo que surge tras la planificación de la transmisión

Tras los análisis efectuados, creemos que la planificación de la transmisión eléctrica es un claro ejemplo de ejercicio unilateral de la potestad de planificación de la Administración. Dicha potestad se materializa a través de diversos actos unilaterales de la Administración, sin que pueda sostenerse que exista una relación contractual entre el Estado y quien ejecuta las obras de expansión.

En efecto, en el marco de la planificación de la transmisión, la Administración fija el contenido de las obras de expansión por medio de la dictación de un decreto supremo. La ejecución de dichas obras es licitada por el Coordinador, quien elige la mejor oferta de entre quienes participan en el proceso concursal respectivo. Sin embargo, los derechos para ejecutar o para ejecutar y explotar la obra planificada (en los casos de ampliación y obras nuevas, respectivamente) solo surgen con la dictación del respectivo decreto del Ministerio de Energía. Así se desprende del art. 122 LGSE, el cual, al regular la posibilidad de entregar en garantía los derechos de ejecución y explotación de un proyecto de transmisión nacional, alude expresamente a "(...) los derechos que para dichas empresas nacen del decreto indicado precedentemente..." [la cursiva es nuestra]. Lo anterior ha sido reconocido recientemente por el D.S. No 167/2019, de Energía, que aclara el Decreto No 418 exento, de 4 de agosto de 2017, del Ministerio de Energía, que fija el listado de las instalaciones de transmisión zonal de ejecución obligatoria, necesarias para el abastecimiento de la demanda. El considerando 18 del citado decreto sostiene que "(...) en virtud de lo señalado en los dos considerandos precedentes, se advierte que los derechos para ejecutar y explotar las obras nuevas o de ampliación en comento nacen o se asignan con los respectivos decretos a los que se refiere el artículo $96^{\circ}$ de la ley, a saber: (i) el que fija los derechos y condiciones de ejecución y explotación de las obras nuevas; y, (ii) el que adjudica la construcción y ejecución de las obras de ampliación. De modo que, la adjudicación que realiza el Coordinador de los derechos de ejecución y explotación del proyecto de obra nueva o para la construcción y ejecución de las obras de ampliación, según corresponda, solo tiene por finalidad identificar las ofertas adjudicadas en el proceso de licitación, pero no generar derechos, ya que, tal como se señaló, éstos nacen una vez perfeccionado el correspondiente acto administrativo...".

Por tanto, como se puede apreciar, la Administración del Estado establece por medio de actos unilaterales cuáles son las líneas o subestaciones que deben ejecutarse a fin de cumplir con la planificación efectuada tanto de manera general (energética) como específicamente respecto de la transmisión. Tales actos de planificación son vinculantes y deben ser ejecutados por medio de mecanismos de competencia que tienen como resultado el otorgamiento ex novo de derechos para ejecutar o ejecutar y explotar las obras en cuestión. 
En dicho contexto, si bien hay un involucramiento por parte de la Administración o, más ampliamente, del Estado por medio del Coordinador Eléctrico Nacional, no es posible afirmar que el mismo pueda dar origen a una relación contractual, i.e., a un contrato administrativo, por cuanto no concurren varios elementos de éste. En efecto:

(i) En la ejecución de las obras de expansión no es posible apreciar la existencia de un sinalagma entre el Estado y quien ejecuta las obras ${ }^{54}$, esto es, prestaciones mutuas y recíprocas ${ }^{55}$, ya que las obras no se ejecutan para el Estado, sino para quién se las adjudica (en el caso de las obras nuevas) o para quién es titular de las obras que se amplían (en el caso de las obras de ampliación). Como se expuso latamente, el rol del Estado en esta materia es determinar las necesidades de expansión de la infraestructura y ordenar su ejecución, fijando los derechos para ello. Pero nada más.

(ii) La ausencia de sinalagma se aprecia, asimismo, en que el Estado no efectúa pago alguno por las obras que se ejecutan, ya que, precisamente, las obras no se ejecutan para él; trátase de obras privadas y no obras públicas. Las obras de expansión se remuneran por los usuarios, esto es, por la deman$\mathrm{da}$, de acuerdo con los VATT o VI ofertados en los casos de obras nuevas y de ampliación, respectivamente (art. 99 LGSE).

(iii) Por último, el Estado no posee atribuciones exorbitantes que permitan sostener la existencia de un desequilibrio posicional como el inherente a los contratos administrativos. En efecto, el Estado no puede variar las obras una vez adjudicadas ni puede poder término a las mismas una vez adjudicadas.

Lo expuesto, desde luego, no es novedoso, sino que se puede apreciar en las denominadas autorizaciones de funcionamiento, en las cuales, dado el involucramiento duradero de la Administración en el marco de la actividad autorizada, en orden a verificar su adecuada ejecución, surge una relación jurídica, i.e., una relación jurídico-administrativa, que en caso alguno posee naturaleza contractual ${ }^{56}$. Por lo expuesto es que parece claro que no es po-

\footnotetext{
54 Se afirma que "(...) el contrato administrativo es una institución 'lógico-jurídica' sinalagmática, comprensible en términos de derecho de las obligaciones...". CONCHA 2012, 52-53.

55 En efecto, se sostiene que "(...) [s]e enfatiza la bilateralidad de los contratos administrativos por ser sinalagmáticos, dado que ambas partes acuerdan en virtud del contrato, obligaciones mutuas y recíprocas, razón por la cual los contratantes tienen el mutuo y recíproco carácter de deudores y acreedores entre sí, al haberse obligado los unos recíprocamente respecto de los otros...". FERnÁNDEZ 2016, 170.

56 Cfr., entre muchos otros, Maurer 2012, 173. En contraposición con el Derecho Civil, desde el ámbito del Derecho Administrativo aún no se efectúa una clara construcción de una teoría general de la relación jurídico-administrativa, dado que ella se ha visto opacada y postergada por los desarrollos realizados respecto de un aspecto particular de la misma (el derecho público subjetivo). Pese a ello, la doctrina ha destacado que la regulación de la relación jurídico-administrativa es precisamente el objeto del Derecho Administrativo, llegando a afirmarse que dicha regulación especial es el Derecho Administrativo. Al respecto vid. García De Enterría y Fernández, 2006, 15; SOto 2009, 219; Moraga 2010, 73-75; MAURER 2012, 175.
} 
sible aludir a la existencia de un contrato administrativo entre el Estado y el ejecutor de obras de expansión.

\section{Conclusiones}

La existencia de infraestructuras en red puede implicar severas afectaciones a la competencia, que justifican la intervención del regulador, con el objeto de resolver o al menos aminorar las fallas de mercado a que ellas pueden dar lugar. Una de las formas de intervención a las que el Estado puede echar mano es la planificación de tales infraestructuras. Por medio de la planificación se busca ordenar la instalación o la expansión de tales instalaciones, de manera eficiente y satisfaciendo asimismo otros fines de interés público que puedan identificarse en las regulaciones propias de cada uno de los sectores sujetos a tal intervención. Para ello, la planificación no prejuzga la existencia de bienes públicos o privados, sino que, a partir de ello arbitra los mecanismos específicos para poder ejecutar tal planificación en los términos indicativos o imperativos que correspondan según la regulación sectorial. Y, asimismo, la planificación puede utilizar tanto instrumentos unilaterales como negociales para amparar las relaciones jurídicas que surjan con ocasión de la ejecución de las infraestructuras en red.

Un claro ejemplo de una intensa actividad de planificación estatal de carácter imperativa es la planificación de la transmisión eléctrica. En ella, el Estado, de forma centralizada, determina cuáles son las infraestructuras que año a año deben ejecutarse o ampliarse, para dar adecuada respuesta a las variables condiciones de demanda existentes. Pero son los particulares los que están llamados a ejecutar tales instalaciones, de acuerdo con los plazos y condiciones dispuestas a tal fin por el regulador, y por medio de procedimientos de asignación concursal que permiten la introducción de competencia en un segmento por esencia monopólico como es la transmisión de energía. En este supuesto, si bien existe una relación permanente entre regulador y regulado -i.e., un involucramiento constante-, ella no posee la naturaleza jurídica de una relación contractual de tipo administrativa, sino que se trata de una relación jurídico-administrativa existente al alero de un acto administrativo unilateral del Estado, tal y como ocurre en las autorizaciones de funcionamiento.

Tales consideraciones son relevantes dado que permiten enmarcar correctamente las diversas dificultades que puedan surgir en relación con la planificación de la transmisión, aportando no solo un marco de análisis, sino que, asimismo, de resolución de las mismas.

\section{Bibliografía citada}

AA.VV. (2010). I nodi delle reti. Infrastrutture, mercato e interesse pubblico. Roma, Passigli Editori, 468 pp.

Aguilar, Óscar (2004). Principios jurídicos aplicables a las infraestructuras públicas. Revista RAP, Organización administrativa, función pública y dominio público, pp. 375-437. 
Alé, Jorge (1990). Un nuevo esquema de regulación de monopolios naturales. Estudios Públicos, $\mathrm{N}^{\circ} 37, \mathrm{pp} .165-226$.

Ariño, Gaspar (2003). Principios de Derecho Público Económico. Modelo de Estado, gestión pública, regulación económica. Madrid, Universidad Externado de Colombia, Fundación de Estudios de Regulación, 829 pp.

ArIÑO, Gaspar (2007). El enigma del contrato administrativo. Revista de Administración Pública, No 172, pp.79-102.

BıCKENBACH, Frank (1999): Regulierung und Wettbewerb im Bereich der Netzinfrastrukturen: Begründung, Regeln und Institutionen, Kiel Working Paper, N 910, Institut für Weltwirtschaft (IfW), Kiel.

Boquera, José María (1985). Derecho Administrativo I. España, Instituto de Estudios de Administración Local, $5^{\mathrm{a}}$ ed., 382 pp.

Caballero, Rafael (2003). Infraestructuras en red y liberalización de servicios públicos. España, Instituto Nacional de Administración Pública, 456 pp.

Concha, Ricardo (2012). Nulidad del contrato de la Administración. Aplicabilidad de las reglas civiles. Chile, Abeledo Perrot, Thomson Reuters, 314 pp.

Cordero, Eduardo (2007). El derecho urbanístico: los instrumentos de planificación territorial y el régimen jurídico de los bienes públicos. Revista de Derecho (Valparaíso), vol. 29, pp. 269-298.

DANós, Jorge (2015). La regulación económica. En: Rodríguez-AranA, Jaime, Rodríguez, Libardo [dir.] y Rodríguez, María del Carmen [coord.], Curso de Derecho Administrativo Iberoamericano, España, Comares, Instituto Nacional de Administración Pública, pp. 559-5712.

De La CRUZ, Juan (2001). Nuevas perspectivas en la regulación de las infraestructuras. En: RDU [eds.], Nuevo derecho de las infraestructuras, España, Editorial Montecorvo, pp. 119-160.

De La CuétARA, Juan (2001). Sobre infraestructuras en red y competencia entre redes. En: RDU [eds.], Nuevo derecho de las infraestructuras, España, Editorial Montecorvo, pp. 197-275.

Diez-PICAzo, Luis (1982). La doctrina del precedente administrativo. Revista de Administración Pública, N 98, pp. 7-46.

Fernández, Jorge (2016). Derecho Administrativo. México, Universidad Nacional Autónoma de México, 563 pp.

Fernández, José (2014). La Constitución Política y el urbanismo. Revista de Derecho Público, Edición Especial, pp. 115-121.

Fernández, María Yolanda (2003). Estatuto Jurídico de los Servicios Esenciales Económicos en Red. España, Instituto Nacional de Administración Pública, 650 pp.

FERRARI, Erminio (2000). I servizi a rete in Europa. Italia, Raffaello Cortina Editore, 406 pp.

Fuentes, Mindy (2015). Análisis legal y doctrinario del Ordenamiento y Planificación Territorial en Chile, características, relación y diferencias. Sustainability, Agri, Food and Environmental Research, vol. 3, N², pp. 12-21.

García De Enterría, Eduardo y Fernández, Tomás-Ramón (2006). Curso de Derecho Administrativo II. España, Thomson Civitas, $10^{\mathrm{a}}$ ed., 752 pp.

Gómez-lbáñez, José (2003). Regulating Infrastructure. Monopoly, Contracts, and Discretion. USA, Harvard University Press, 448 pp.

Houston, G., y JOHnson, C. (2006). EPRI-GTC Overhead Electric Transmission Line Siting Methodology. Disponible en: <https://bit.ly/2LHwnmH>, [fecha de consulta: 8 julio 2019].

LEAL, Brigitte (2015). Planificación, colaboración y autocontrol: nuevos instrumentos normativos para la administración fiscalizadora. Revista de Derecho Público, Vol. 83, $2^{\circ}$ sem., pp.105-122.

LeAL, Brigitte (2015a). La Potestad de Inspección de la Administración del Estado. Chile, Cuadernos del Tribunal Constitucional, N 56, 297 pp.

LÓPEZ-JuRAdo, Francisco (2009). Técnicas específicas de los servicios en red", en: Muñoz, Santiago, y EsteVE, José [dirs.], Derecho de la Regulación Económica, I, Fundamentos 
e Instituciones de la Regulación, España, lustel, Fundación Instituto Universitario de Investigación José Ortega y Gasset, pp.759-825.

MANACORDA, Paola y De VINCENTI, Claudio (2010). I nodi delle reti. Infrastrutture, mercato e interesse pubblico, Italia, Passigli Editori, 455 pp.

Mankiw, Gregory (2012). Principios de Economía. México, Cengage Learning Editores, $880 \mathrm{pp}$.

MANTECA, Víctor (2011). Las potestades administrativas: organización, planificación y autotutela. Disponible en: <https://bit.ly/2xv2fmi> [fecha de consulta: 3 julio 2019].

Martínez, Daniela (2017). Hacia un nuevo régimen de ordenamiento territorial: desafíos para el sector energético. Revista de Derecho Administrativo Económico, No 24, pp. 103-117.

Martín-Retortillo, Sebastián (1966). Presupuestos políticos y fundamentación constitucional de la planificación administrativa. Revista de Administración Pública, №50, pp. 111-146.

Martín-Retortillo, Sebastián (1988). Derecho Administrativo Económico. España, La Ley, TI, 504 pp.

MaUReR, Hartmut (2012[2006]). Derecho Administrativo Alemán (trad. María José Bobes Sánchez, María Mercé Darnaculleta I Gardella, José García Alcorta, Javier García Luengo, Alejandro Huergo Lora, Núria Magaldi, Oriol Mir Puigpelat, Marc Tarrés Vives y Gabriel Doménech Pascual). México, Universidad Nacional Autónoma de México, 578 pp

MAYER, Otto (1951). Derecho Administrativo Alemán. Argentina, Editorial Depalma, Tomo I, Parte Especial, $319 \mathrm{pp}$.

Ministerio de Energía (2018). Guía de orientación para los estudios de franjas de transmisión eléctrica. Chile, Gobierno de Chile, 120 pp.

Ministerio de Energía, Ministerio del Medio Ambiente y Servicio de Evaluación Ambiental (2018). Guía para articular el proceso de evaluación ambiental estratégica con la evaluación de impacto ambiental. Estudios de franjas y proyectos de transmisión eléctrica. Chile, Gobierno de Chile, $50 \mathrm{pp}$.

MONTERO, Juan José (2016). Regulación Económica. La actividad administrativa de regulación de los mercados $2^{\mathrm{a}}$ ed. (Tirant lo Blanch, España).

Moraga, Claudio (2010). Tratado de Derecho Administrativo, La Actividad Formal de la Administración del Estado. Chile, Abeledo Perrot, LegalPublishing, T VII, 574 pp.

Moraga, Claudio (2016). Sobre la regulación (Apuntes de Clase). Chile, Facultad de Derecho, Universidad de Chile.

Muñoz, Santiago (2015). Tratado de Derecho Administrativo y Derecho Público General, La Actividad Regulatoria de la Administración. España, Agencia Estatal Boletín Oficial del Estado, vols. XIV, $4^{\mathrm{a}}$ ed., $5600 \mathrm{pp}$.

OelCKers, Osvaldo (1984). Nuevos roles de la Administración Pública y su regulación por el Derecho Administrativo. Revista Chilena de Derecho, Vol.11, pp.471-477.

Pallavicinı, Julio (2015). Derecho Público Financiero. Chile, Thomson Reuters, La Ley, $382 \mathrm{pp}$.

PARADA, Ramón (2010). Derecho Administrativo III. Bienes públicos. Derecho urbanístico. España, Marcial Pons, $12^{\mathrm{a}}$ ed., $432 \mathrm{pp}$.

Pérez, Antonio (1998). La ordenación del territorio, una encrucijada de competencias planificadoras. Revista de Administración Pública, № 147, pp. 97-138.

RASCHAUER, Bernhard (2009). Allgemeines Verwaltungsrecht. Austria, Springer, $3^{a}$ edición, $458 \mathrm{pp}$.

SCHMIDT-AsSMANN, Eberhard (2003). La teoría general del derecho administrativo como sistema. España, Instituto Nacional de Administración Pública, Marcial Pons, 475 pp.

SebASTIANI, Mario (2010). Le infrastrutture di trasporto: programmazione, concorrenza, interesse pubblico. En: MANACORDA, Paula [ed.], I nodi delle reti. Infrastrutture, mercato e interesse pubblico, Italia, Passigli Editori, pp. 47-156.

Sото, Eduardo (2009). Derecho Administrativo. Temas Fundamentales. Chile, LegalPublishing, $788 \mathrm{pp}$.

Verdugo, Mario, Pfeffer, Emilio y Noguelra, Humberto (2005). Derecho Constitucional. Chile, Editorial Jurídica, TI, $2^{\mathrm{a}}$ ed., 375 pp. 
VETRO, Francesco (2005). Il servizio pubblico a rete. L'esempio paradigmático dell'energia elettrica. Italia, Giappichelli, 388 pp.

VILLAR, José (1999). Derecho Administrativo Especial. Administración Pública y Actividad de los Particulares. España, Ed. Civitas, 435 pp.

\section{Normas citadas}

D.F.L. No 323, de 1931, Ley de Servicios de Gas, Diario Oficial de 30 de mayo de 1931.

D.L. N ${ }^{\circ} 1.263 / 1978$, Ley Orgánica de Administración Financiera del Estado, Diario Oficial de 28 de noviembre de 1975.

D.F.L. N 458, de 1975, Ley General de Urbanismo y Construcción, Diario Oficial de 13 de abril de 1976.

Ley $N^{\circ}$ 18.168, Ley General de Telecomunicaciones, Diario Oficial de 2 de octubre de 1982

D.F.L. No 382, de 1988, Ley General de Servicios Sanitarios, Diario Oficial de 21 de junio de 1989.

D.F.L. $N^{\circ} 1 / 19.653$, fija el Texto Refundido, Coordinado y Sistematizado de la Ley N 18.575, Orgánica Constitucional de Bases Generales de la Administración del Estado, Diario Oficial de 17 de noviembre de 2001.

D.F.L. N 1 1-19.175, de 2005, fija el Texto Refundido, Coordinado, Sistematizado y Actualizado de la Ley No 19.175, Orgánica Constitucional sobre Gobierno y Administración Regional, Diario Oficial de 8 de noviembre de 2005.

D.F.L. $N^{\circ} 4 / 20.018$, fija el Texto Refundido, Coordinado y Sistematizado del D.F.L. No 1, del Ministerio de Minería, de 1982, Ley General de Servicios Eléctricos en materia de Energía Eléctrica, Diario Oficial de 5 de febrero de 2007.

Ley N ${ }^{\circ} 20.936$, Establece un Nuevo Sistema de Transmisión Eléctrica y Crea un Organismo Coordinador Independiente del Sistema Eléctrico Nacional, Diario Oficial de 20 de julio de 2016.

Ley $N^{\circ} 21.074$, Fortalecimiento de la Regionalización del País, Diario Oficial de 15 de febrero de 2018.

\section{Jurisprudencia citada}

Sentencia TC Rol No 2.751-14-INA: Tribunal Constitucional, 7 de julio de 2015.

Dictamen No 1 (2013): Panel de Expertos, 14 de marzo de 2013.

Dictamen $N^{\circ} 7$ (2018): Panel de Expertos, 10 de agosto de 2018.

Dictamen Nº 36.448 (2016): Contraloría General de la República, 17 de mayo de 2016. 